\title{
From single to double track: effects of alternative extension measures
}

\author{
O. Lindfeldt \\ Vectura Consulting AB, Stockholm, Sweden
}

\begin{abstract}
Extension of single-track lines into double-track will be an important part of future rail investments in Sweden. Most Swedish railway lines are single-track and the steady growth in demand for passenger and freight transportation calls for more capacity, which in turn imposes a need for more double-track sections.

This paper points out three minor measures that can be taken on single-track lines before they are completely extended into double-tracks: additional crossing loops, extended crossing loops to enable parallel entrance, and partial doubletracks. A generic model for timetable analysis, TVEM (Timetable Variant Evaluation Model), is applied to evaluate the effect of the three measures through generation of 20,000-50,000 alternative timetables.

The evaluation shows that none of the measures significantly affect the maximum capacity, i.e. the number of trains in the best timetable alternative. This indicates that the longest inter-loop distance, has to be substantially shortened through either an additional crossing loop or a partial double-track. A parallel entrance facility on a crossing loop does not affect the time distance between adjacent loops so much that it can be considered to increase maximum capacity.

However, all three measures result in higher timetable flexibility, i.e. number of timetable variants for a given number of trains (capacity level). Partial doubletracks give much more timetable flexibility than additional crossing loops and parallel entrance facilities on crossing loops.

It is reasonable that higher timetable flexibility imposes a higher practical, realizable capacity, since a line section that can be scheduled flexibly is less likely to constrain the overall capacity on the entire line.

Keywords: single-track, double-track, crossing loop, scheduling, railway capacity.
\end{abstract}




\section{Introduction}

The Swedish railway network consists of a number of single-track lines and a few double-track lines (see figure 1). Two main lines are quadruple-track on short sections close to Stockholm. The predominance of single-track lines makes the railway system unattractive to passengers and freight transport customers, due to low capacity, low average speeds and very frequent delays.

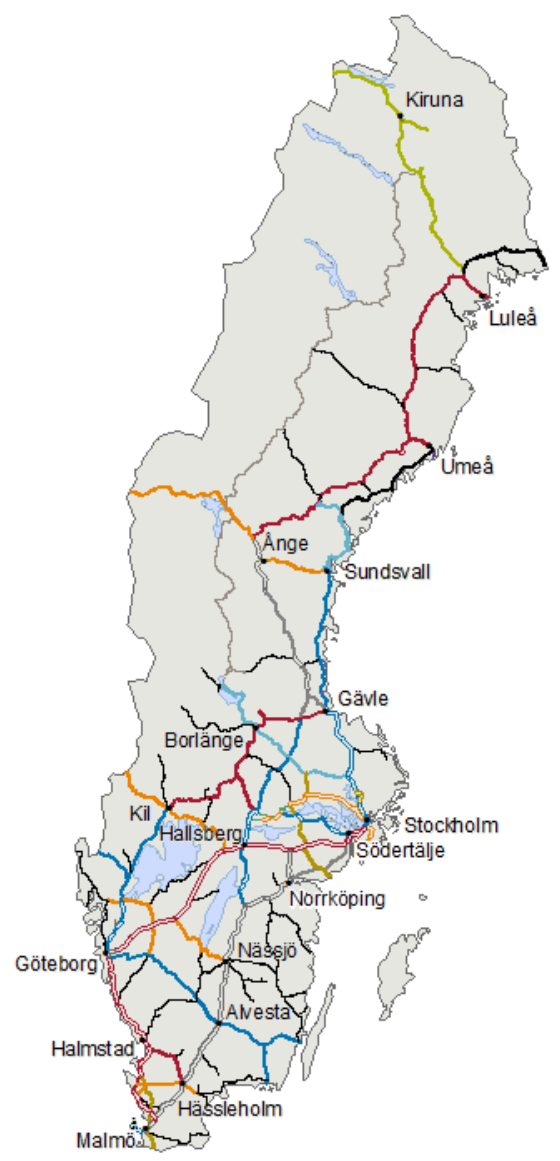

Figure 1: The Swedish railway network, double-track lines indicated with double lines.

These facts make the single-track part of the network a major challenge for the development of the Swedish railways. The conversion of single-track lines to double-track is a time-consuming and expensive process. Nor is full conversion from single- to double-track always motivated, simply because the capacity demand is not high enough to motivate a double-track line. 
This calls for less extensive measures where the operational properties of a single-track line are improved through different complements and adjustments in the infrastructure. The bi-directed traffic and the crossings that it induces are the main limitations of single-tracks. This means that measures that limit the negative effects of crossings are feasible as intermediate steps before extension into double-track. Some examples of such measures are:

- Additional crossing loops

- Extended crossing loops for parallel entrance

- Partial double-tracks

- Additional block sections (these do not affect the crossings but make bunched operation more efficient).

This paper concerns the capacity effect of the measures listed above. The measures and their expected effects are described in section 2. A method for atomised timetable analysis (TVEM) is described in section 3 and in section 4 the method is applied on a heavily utilised Swedish railway line. Everything is summed up in section 5 and some important conclusions are drawn.

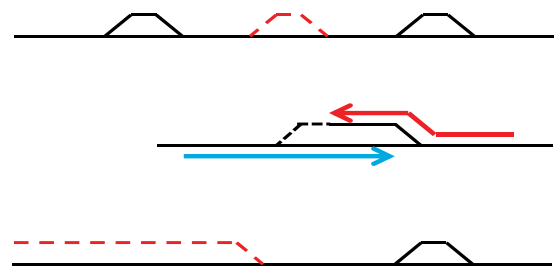

Figure 2: Additional crossing loops, parallel entrance and partial doubletracks.

\section{Possible extension measures and expected effects}

Some important capacity-increasing measures were listed in the previous section. The first one is obvious. Adding more crossing loops increases capacity since the time between two trains in opposite directions can be shortened when the time distance between the crossing loops decreases. The effect analysis of additional crossing loops can be kept simple as long as only the local effect is concerned. It is much more complicated to perform a system analysis and find the effect of one or a few added crossing loops on an entire railway line.

This problem is partly solved if timetable analysis is applied. Timetable construction is, in some sense, an indirect capacity calculation. The maximum capacity is found when no more trains can be fitted into the timetable. The main advantage of the timetabling method is that all, or at least most, system effects are caught. One important example is the fact that a new crossing loop is not useful for all kinds of timetable structures and timetable strategies - there is an interaction between infrastructure and timetable, a system effect that is difficult to catch without pure timetable construction! 


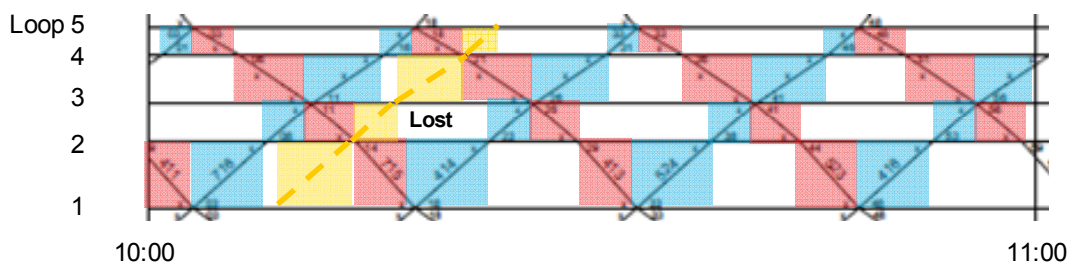

Figure 3: Blocking diagram for a single-track line. An additional train can be added every 15 minutes (yellow dashed line) but this results in lost capacity that cannot be used for any other train.

About $50 \%$ of the Swedish crossing loops do not allow parallel entrance, which means that both entrance signals cannot show clear aspect at the same time. In case of a simultaneous approach, one of the trains has to wait at its entrance signal until the crossing train is securely stopped on the loop track. In practice this means that the arrivals of crossing trains have to be time-separated in the timetable so that the first train arrives at least 3 minutes before the second one (see figure 4). In case of parallel entrance, the prescribed time separation is only 1 minute.

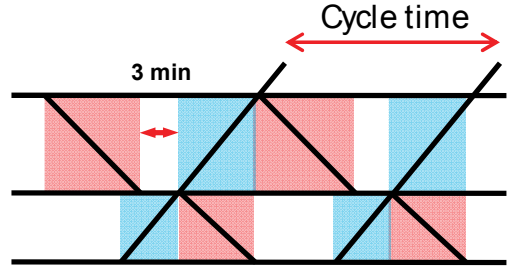

No parallel entrance allowed

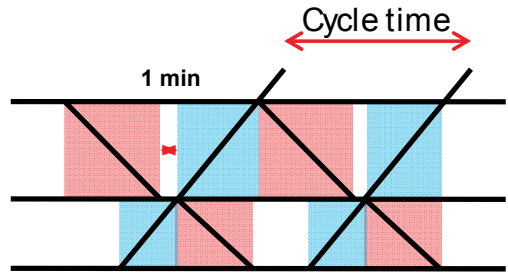

Parallel entrance allowed

Figure 4: $\quad$ Effect of parallel entrance at crossing loops.

Adjusting a crossing loop for parallel entrance means that the loop tracks are extended about $200 \mathrm{~m}$, which is the required safety distance between the intermediate signal at the end of the loop track and the point where a collision may occur if one of the trains does not stop properly. The adjustment also involves changes in the interlocking, which means that the total adjustment is rather extensive.

The effect of parallel entrance is heavily dependent on the timetable. The best case is shown in figure 4 where the parallel movement facility is used over and over again, which gives a shorter cycle time for a periodic timetable. The other extreme is when different kinds of timetable constraints, from the surrounding infrastructure, imply that the parallel movement cannot be utilised. This can be the case if the timetable cannot be tightened so that the crossing trains actually 
arrive with less than 3 minutes time separation, or if the crossing loop is simply not used for crossings. Altogether, this means that timetable construction and analysis seems to be a feasible method for this measure as well.

A third, "intermediate", measure on the way to a complete double-track is partial conversion through the construction of partial double-tracks. In some sense, the effect of partial double-tracks is even more difficult to describe and evaluate, since it results in a very high, but local, capacity increase. This means that the remaining single-track sections must be more heavily utilised if more trains are to be operated. Making such an increase in utilisation possible is exactly the objective of a partial double-track. Timetable analysis has also proven to be efficient for capacity evaluation of partial double-tracks.

The fourth measure, additional block sections, is also an important way to increase capacity on single-track lines. Additional block sections are very feasible for bunched operation, which occurs on some of the most heavily utilised single-track lines in Sweden. One example is the "Freight line through Bergslagen" where the stream of freight trains has one direction during the evening and the opposite direction during the late night and early morning hours (see [1]).

Another important feature of additional block sections is that they require only minor investment compared to additional crossing loops, extended crossing loops and partial double-tracks. This means that additional block sections are in many cases really beneficial measures with great effects for fairly limited investment cost.

Timetable construction and analysis is also useful for effect evaluation of additional block sections. However, the timetables used in the analysis must reflect the bunch patterns that are likely in the future, which means that the sampling of train paths has to be adjusted according to this (see [1] for details).

This short article will focus on the first three measures: additional crossing loops, extended crossing loops for parallel entrance movements and partial double-tracks.

\section{Method for capacity evaluation}

Timetable construction is a fairly straightforward method for capacity analysis. It has proven to be a very useful method since it gives insights in the properties of the infrastructure, the timetable and the interaction between them. This means that timetable analysis gives more detailed results than the UIC Code 406 compression method that is usually used in strategic planning in Sweden [2].

The weaknesses and limitations of the UIC Code 406 are shown in [3], i.e. the dependence on the existing timetable, the question of how to treat scheduled delays in a proper way and the difficulties in analysing entire lines consisting of both line sections and station and loop tracks.

Timetable analysis, with several thousand alternative timetables, means that not only today's timetable, but also a great many possible future timetables can be analysed. By definition a timetable analysis includes both line sections and loops and stations. The entire railway line is thus analysed synchronously. 
The scheduled delay is easy to keep track of throughout the timetable analysis procedure since it simply determines which train paths to accept and which to reject (see $[4,5]$ for further discussions about scheduled delays).

The number of possible timetables for a given infrastructure is infinite. This means that the analyst has to limit the number of analysed timetables in some way. One way of doing this is to utilise the fact that most passenger traffic is cyclic, with a timetable that repeats every second hour or every hour to limit the number of timetable variants. This is exactly how TVEM, Timetable Variant Evaluation Model, works.

The idea of TVEM is to evaluate an infrastructure design with respect to a required traffic mix. Both the infrastructure and the parameters that define the traffic may appear as variables. A more detailed description of the model is given in $[6,7]$ and $[1]$. This article includes only a brief model description.

Capacity on lines with mixed traffic depends on many parameters. In TVEM the parameters of greatest interest for Swedish circumstances have been chosen as variables and put into three groups, see the left side of figure 5. Just as in ordinary timetable construction, the infrastructure is modelled with running times and time supplements for the vehicles that are used for the trains.

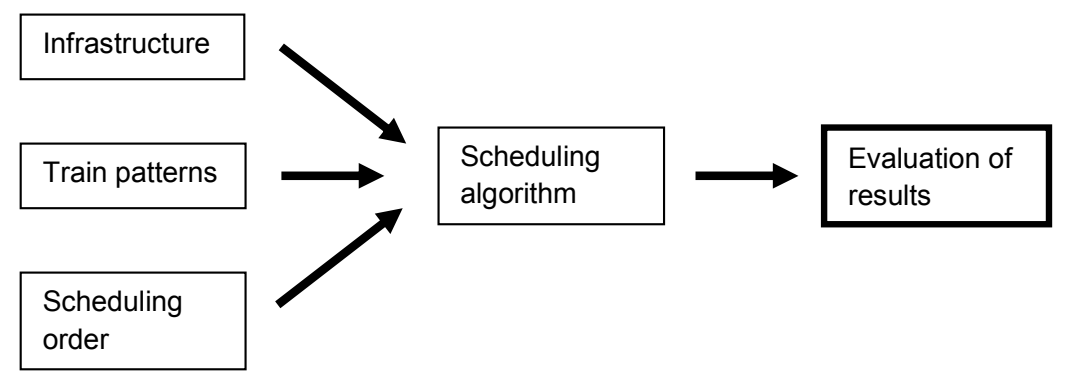

Figure 5: $\quad$ Structure of TVEM.

The traffic is defined by train patterns. A typical single-track line may be operated by 4-6 different patterns, e.g. high-speed, regional and freight trains in two directions. Each train pattern is given attributes such as vehicle type, frequency of service (for cyclic patterns), stopping pattern, utilisation restrictions for loop tracks, etc.

A final input of great importance is the scheduling order. This shows the order in which the train patterns are to be scheduled (priority order). The nonperiodically operated patterns (freight trains), which lack frequency, are by definition scheduled last.

Given the input described above, the algorithm is a straightforward procedure where a great number of timetables that satisfy the assumptions are tested. This is possible since the number of timetable variants is limited as long as there are few train patterns and the frequency of service is high.

Figure 6 shows the three major steps in the scheduling algorithm. The highest prioritised pattern, most often the most important passenger traffic pattern, is scheduled first. The trains in this pattern do not interact with any other trains. 


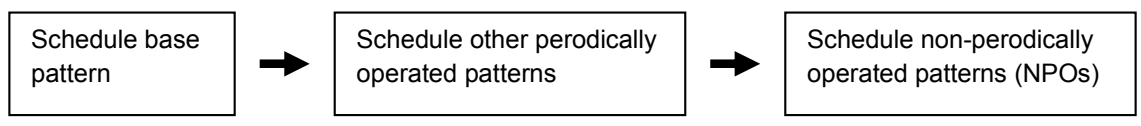

Figure 6: Major steps in TVEM's scheduling algorithm.

They are scheduled in an empty timetable. This pattern is then kept fixed and the timetable variants are instead created by the locations of the other patterns.

The next step means scheduling of the following periodically operated patterns. These are introduced in the timetable one after the other according to the scheduling order. During the scheduling, each additional train is adjusted as necessary against previously scheduled trains by introducing crossings, overtaking and bending (catch-up situations). The scheduling procedure follows general principles (blocking time theory) for timetable construction (see [8]).

The last major step is to schedule the non-periodic operated patterns. Trains within these patterns are handled individually, train by train, according to a predefined or randomised scheduling order.

Two processes in this scheduling algorithm give rise to timetable variants:

- $\quad$ Systematic shift of periodically operated patterns.

- $\quad$ Different scheduling orders of individual trains within NPO patterns, i.e. sampled replicates.

The algorithm is designed to step forward through all patterns to find feasible timetable solutions. This means that alternative time shifts for each periodically operated pattern are sought and stored. Only previously scheduled patterns affect these alternative shifts. Once the alternative time shifts for a pattern have been found, the algorithm realises the first time shift and schedules the pattern according to this time location. A copy of this timetable is then stored to be used as the starting point for scheduling the following pattern.

Data is collected for the timetable variant as soon as all patterns have been successfully scheduled. Failure to schedule a pattern results in a backward step to the previous pattern, which is then scheduled according to the succeeding time shift stored in the initial search.

\section{Application - upgrade of a Swedish single-track line}

The single-line section between the cities of Falun and Borlänge in central Sweden is one of the most heavily utilised lines in the country with about 85 trains/day. The line section is only $25 \mathrm{~km}$ long and has two crossing loops, neither of which is long enough to allow parallel entrance for crossing trains. The line is to be converted to double-track some time in the future, but lack of financial resources means that minor measures are needed before any major investment can be made.

Table 1 shows five possible measures to increase capacity on the line. The measures are also shown schematically in figure 7. Alternatives 1 and 2 include measures that are very common on single-track lines, whereas alternatives 3-5 include partial double-track sections, which are actually part of the future double-track conversion. 
Table 1: $\quad$ Evaluated alternatives.

\begin{tabular}{l|l}
\hline Alternative & \multicolumn{1}{c}{ Measure } \\
\hline Existing line & None \\
1 & Extended crossing loops for parallel entrance facility \\
2 & Alternative 1 plus one additional crossing loop \\
\hline 3 & Partial double-track in the east end \\
4 & Partial double-track in the west end \\
5 & Partial double-track in east and west end \\
\hline
\end{tabular}

\section{Borlänge Ornäs Hinsnoret Falun}

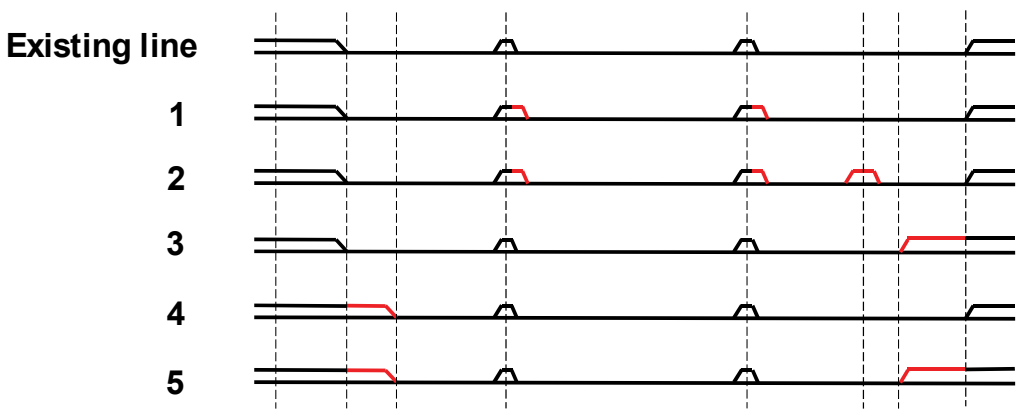

Figure 7: Alternative measures for capacity increase (red). The existing line has two crossing loops; Ornäs and Hinsnoret.

The main questions are how the five alternatives affect the capacity of the line section and whether any of the double-track alternatives is better than the two alternatives that focus on the crossing loops. These questions give a unique opportunity to compare alternative strategies and the results will be useful on several lines around Sweden.

The traffic on the line can be divided into six independent train patterns (see table 2). The traffic is quite homogeneous, due to some sharp curves that limit the speed. Patterns 1-4 are passenger trains that are operated periodically. The frequency is here set to one train every 80 minutes, which is a mean value for the 14 trains that are to be operated within each pattern during 17 hours each day. Up- and down-bound freight trains are operated non-periodically, which means that these trains are scheduled individually train by train.

TVEM was applied to determine the capacity for each of the five alternatives and the existing line. One of the IC train patterns was used as base pattern, i.e. scheduled first and not affected by any other trains. This pattern was followed by patterns 2-4 in order. The model generates the timetable variants through systematic shifts of the relative time locations for these patterns. Ideally, there are 80 alternative time shifts for each of the patterns, which gives $80^{3}=512,000$ alternative timetables just for the periodically operated traffic. However, 
Table 2: $\quad$ Train patterns. The number of trains within pattern 5 and 6 depends on the relative location of the passenger train paths, i.e. the timetable.

\begin{tabular}{l|l|l|l|l|l}
\hline Pattern & $\begin{array}{l}\text { Type of } \\
\text { train }\end{array}$ & Direction & $\begin{array}{l}\text { Nominal } \\
\text { run time } \\
{[\mathrm{min}]}\end{array}$ & $\begin{array}{l}\text { Number } \\
\text { of } \\
\text { trains }\end{array}$ & $\begin{array}{l}\text { Frequency of } \\
\text { service }\end{array}$ \\
\hline $\mathbf{1}$ & IC & Falun - Borlänge & 18 & 14 & $1 / 80 \mathrm{~min}^{-1}$ \\
$\mathbf{2}$ & IC & Borlänge - Falun & 18 & 14 & $1 / 80 \mathrm{~min}^{-1}$ \\
$\mathbf{3}$ & Regional & Falun - Borlänge & 16 & 14 & $1 / 80 \mathrm{~min}^{-1}$ \\
$\mathbf{4}$ & Regional & Borlänge - Falun & 16 & 14 & $1 / 80 \mathrm{~min}^{-1}$ \\
$\mathbf{5}$ & Freight & Falun - Borlänge & 18 & & Non-periodic \\
$\mathbf{6}$ & Freight & Borlänge - Falun & 23 & & Non-periodic \\
\hline
\end{tabular}

headway restrictions reduce the variability to $75 * 70 * 65=341,250$ variants, if the minimum headway is assumed to be 5 minutes.

TVEM makes use of three methods to resolve conflicts between trains:

- Crossing

- Overtaking

- Bending of train paths.

The occurrence of conflicts further reduces timetable variability. Only 20,000-48,000 variants can be found by the model when all conflicts have been resolved according to the acceptance levels for scheduled delay.

Finding a feasible timetable for the passenger trains is only the first model step. In a second step, capacity is determined through a filling process where the spare capacity in-between the passenger trains is filled with freight trains that are scheduled individually. The process terminates when no additional freight train (pattern 5 or 6 ) can be added to the timetable.

Figure 8 summarizes the results. The horizontal axis shows the total capacity, i.e. the $4 * 14=56$ passenger trains plus the number of freight trains that could be scheduled between the passenger trains. The vertical axis shows the number of timetable variants with a capacity equal to or higher than the given capacity.

Figure 8 gives a very clear picture of the effect of the analysed investment alternatives. A remarkable result is that all alternatives have the same maximum capacity. About 128-130 trains/day can be scheduled if the timetable is fully adjusted and optimised for the studied line section. This means that none of the measures affect the real bottle-neck significantly.

One hypothesis is that the maximum capacity is limited by the longest section Hinsnoret - Ornäs. The higher capacity value, 132 trains/day, comes from alternatives 1 and 2 where this section is operationally shortened for crossing trains by the parallel movement facility at the crossing loops. This means that a significant capacity increase requires either a longer double-track section or an additional crossing loop that divides the long inter-loop distance between Hinsnoret and Ornäs into two. 


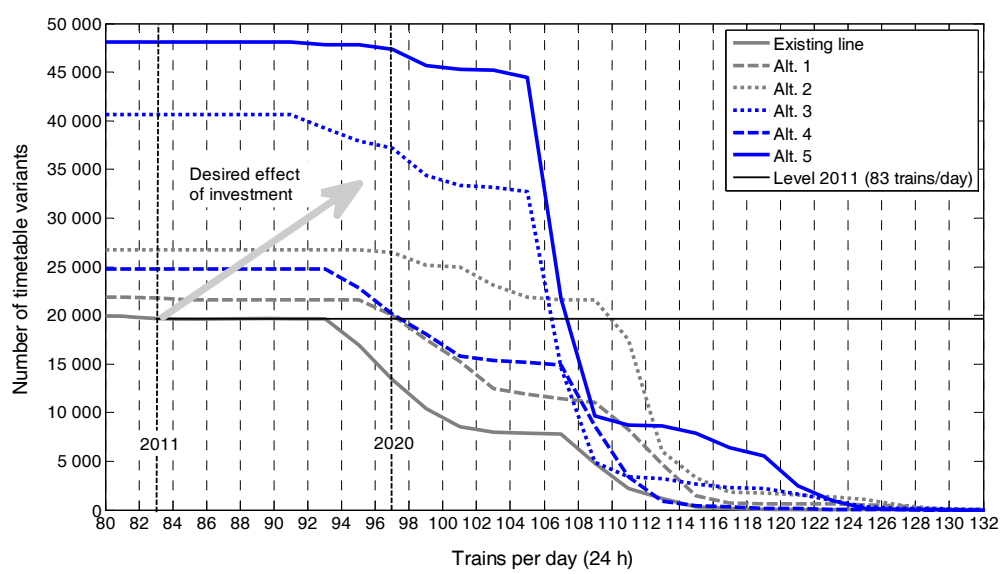

Figure 8: Number of timetable variants with a specific capacity (x-axis) or higher.

Even if the maximum capacity does not differ very much between the alternatives, practical capacity may differ significantly due to differences in the timetable flexibility. This flexibility is very important since it affects the practical capacity, i.e. the capacity that can be realised when the line section is part of a larger network of line sections. The double-track alternatives give much higher timetable flexibility since the two traffic directions are less dependent on each other when crossings can take place on the double-track sections. The grey arrow indicates that the most efficient investment would increase both capacity (number of available train paths) and timetable flexibility for a given capacity level (number of available timetable variants).

Figure 8 also shows that the existing line can handle another 10 trains per day on the current level of timetable flexibility. A more extensive traffic increase requires any of the studied infrastructure measures.

The alternatives can be divided into two groups according to their capacityincreasing effects. Alternatives 1 and 4, i.e. extension of existing crossing loops for parallel movement and a double track on the western part of the line section, gives a lower effect, whereas the other three alternatives result in a much higher capacity increase for the same number of timetable variants.

\section{Conclusions}

This paper points out some important measures to increase capacity on singletrack railway lines: extension of crossing loops to enable parallel entrance for crossing trains, additional crossing loop to reduce the capacity-limiting section and different variants of partial double-tracks. These measures are combined into 5 infrastructure alternatives that are evaluated. 
A model for timetable analysis, TVEM (Timetable Variant Evaluation Model), is applied for the capacity analysis where between 20,000 and 50,000 alternative timetable structures are generated for each of the studied infrastructure designs. The timetable analysis method has several advantages since it captures the interaction effects between different line sections (inter-loop distances).

All tested infrastructure measures were shown to increase timetable flexibility, i.e. number of available timetable structures for a given capacity level (number of trains). Partial double-tracks, which reduce the length of one or several single-track line sections, result in a large increase in timetable flexibility, whereas loop measures such as extended crossing loops and/or additional crossing loops only resulted in a minor increase in timetable flexibility. This is reasonable since a double-track gives much more freedom to schedule crossings, which means fewer restrictions in the scheduling process.

Unfortunately, none of the measures resulted in a significant increase in maximum capacity, i.e. number of trains in best timetable alternative. This indicates that maximum capacity can only be affected if significant measures are taken on the longest inter-station distance. This means that a parallel entrance facility on the crossing loops does not affect the time distance between adjacent loops so much that it can be considered to increase maximum capacity.

The value of increased timetable flexibility is not obvious. It can be argued that high flexibility implies better possibilities to find a feasible timetable for a network of line sections with limited capacity. More research is needed to find out how timetable flexibility affects practical (realisable) capacity. One way of doing this is to analyse a longer line section with more loops and to perform the analysis on alternative configurations.

\section{References}

[1] Lindfeldt, O., 2011. Analysis of capacity on single-track railway lines. In: Hansen, I.A., Wendler, E., Ricci, S., Longo, G., Rodriguez, J. (eds.), Proceedings of $4^{\text {th }}$ International Seminar on Railway Operations Modelling and Analysis, Rome, Italy.

[2] Wahlborg, M., 2004. Banverket experience of capacity calculations according to the UIC capacity leaflet. In: Allan, J., Brebbia, C.A., Hill, R.J., Sciutto, G., Sone, S. (eds.), Computers in Railways IX, pp. 665-673, WIT Press, Southampton.

[3] Lindner, T., 2011. Applicability of the analytical UIC Code 406 compression method for evaluating line and station capacity. Journal of Rail Transport Planning and Management, 1, pp. 49-57.

[4] Kosonen, T., 2010. A method for the improvement need definition of large, single-track rail network analysis and infrastructure using "Rail Traffic System Analysis. In: Ning, B., Brebbia, C. A., Roberts, C., Rumsey, A.F., Sciutto, G., Tomii, N. (eds.), Computers in Railways XII, WIT Press, Southampton. 
[5] Landex, A., 2010. Computation and evaluation of scheduled waiting times for railway networks. In: Ning, B., Brebbia, C. A., Roberts, C., Rumsey, A.F., Sciutto, G., Tomii, N. (eds.), Computers in Railways XII, WIT Press, Southampton.

[6] Lindfeldt, O., 2011. An analysis of double-track railway line capacity. Transportation Planning and Technology, 34(4), pp. 301-322.

[7] Lindfeldt, O., 2010. Railway operation analysis. Evaluation of quality, infrastructure and timetable on single and double-track lines with analytical models and simulation. Royal Institute of Technology. Stockholm. Thesis $(\mathrm{PhD})$.

[8] Pachl, J. 2008. Timetable design principles. In: Hansen, I.A., Pachl, J., eds. Railway timetable and traffic. Hamburg: Eurailpress, Ch. 2, pp. 9-42. 